\title{
Criterios para desarrollo de fortalezas tecnológicas en la UNMSM. Avances para un modelo de investigación emprendedor en ingeniería de procesos
}

\section{RESUMEN}

El estudio se refiere al diseño de la ventaja competitiva de un potencial Parque Científico-Tecnológico en la Facultad de Ingeniería Industrial de la Universidad Nacional Mayor de San Marcos considerando las fuentes de creación de competitividad a fin de aprovechar el potencial de investigación y desarrollo en la UNMSM para generar innovación en contacto con la comunidad empresarial. Para que el Parque CientíficoTecnológico o PCT realice un valioso aporte al conocimiento y la investigación, se ha de sustentar en estrategias y políticas de desarrollo que se orienten a la creatividad y la innovación en productos tecnológicos, incubadora de tecnologías y productos de avanzada, centro de intercambio de oportunidades de desarrollo, planteando criterios para el emprendimiento de negocios. El proyecto PCT como parte de un proyecto integral de creación de ventajas competitivas tecnológicas debe generar expectativas e interés, el proyecto ha de tener alcance geográfico local y descentralizado, buscando un mayor espacio de influencia. Su implantación significaría una valiosa línea de acción de la UNMSM en su proyección a la comunidad y en su contribución al desarrollo del país, con lo cual mantendría su sitial de principal centro académico y de investigación del país.

Palabras clave: parque científico-tecnológico, diamante de desarrollo, modelo de negocio, estrategia competitiva

CRITERIA FOR DEVELOPING TECHNOLOGICAL STRENGTHS in SAN Marcos UNIVERSiTy. AdVANCES For a MODEL OF ENTREPRENEURIAL RESEARCH IN PROCESS ENGINEERING

ABSTRACT

The study overall objective is the design of competitive advantage of Science and Technology Park plan, leaded by the Faculty of Industrial Engineering of San Marcos Major National University, considering sources of creating competitiveness to leverage the potential of research and development in UNMSM for innovation deployment in contact with the business community. For that Science and Technology Park or PCT makes a valuable contribution to knowledge and research, it has to support strategies and policies of creativity and innovation in technology products, technology incubator and new products, advanced clearinghouse oriented to development opportunities, raising the criteria for the business venture and entrepreneurship. The project PCT must generate expectations and interest in the academic and entrepreneurial environment for effective implementation, the project must have local and decentralized geographic scope, seeking greater influence space. Its implementation shall mean a valuable line of action of UNMSM in its outreach to the community and its contribution to national development, which would keep his position as main research and academic center of the country.

Keywords: science and technology park, diamond development, business model, competitive strategy

\section{INTRODUCCIÓN}

El objetivo general es plantear los criterios básicos para crear ventajas competitivas de un potencial Parque CientíficoTecnológico (PCT), ubicado en el contexto de los escenarios prospectivos de desarrollo de la Facultad de Ingeniería Industrial. El estudio competitivo de un PCT se sustenta en el avance teórico del concepto de estrategia y competitividad en industrias, sectores y clusters, en las condiciones vigentes para el desarrollo de las naciones, en el aporte de la academia en la investigación empresarial y en la aparición de las reglas innovadoras y de ruptura de la nueva economía.

Los parques científico-tecnológicos se consolidan en últimos cincuenta años, con diferentes denominaciones como centros de alta tecnología, polos tecnológicos, polos de desarrollo, nodos de innovación, cluster del conocimiento [2], aunque con dos características comunes: concentración espacial-geográfica de actores y redes de contacto e intercambio.

El problema se plantea con la pregunta: “ ¿Cómo son las ventajas competitivas de la Facultad de Ingeniería Industrial en referencia a aprovecharlas mediante un proyecto de parque científico tecnológico?". Se ha desarrollado una investigación empírica a fin de obtener criterios que permitan delimitar las ventajas competitivas que conllevan la propuesta académica hacia en entorno empresarial y social, la identificación del potencial de investigación e innovación mediante el delineamiento de las fuentes de competitividad, de manera que represente el diferencial para el desarrollo que se ha de ofrecer a la comunidad académica y comunidad empresarial.

\section{METODOLOGÍA DE INVESTIGACIÓN}

El estudio es una investigación empírica desarrollada a partir de estudio previo sobre el concepto de parques científicos tecnológicos y las diferentes experiencias de implantación.

\footnotetext{
* Doctor en Ingeniería Industrial, Magíster en Ingeniería Industrial, Ingeniero Industrial, Facultad de Ingeniería Industrial-UNMSM. E-mail: orestescachay@yahoo.es, orestes.cachay@industrial.unmsm.pe

** Doctor en Ingeniería Industrial, Magíster en Administración, Ingeniero Industrial, Economista. Facultad de Ingeniería Industrial-UNMSM. E-mail: aacevedo@speedy.com.pe, aacevedo@industrial.unmsm.pe

*** Magíster en Dirección de Empresas, Ingeniero Industrial, Abogada. UPG Facultad de Ingeniería de Sistemas e Informática UNMSM. E-mail: klinares@speedy.com.pe
} 
Se plantea una investigación cualitativa para la identificación y diseño de la estrategia para implantar un PCT que generar fortalezas a la universidad. Luego se plantea la investigación cuantitativa partir de las respuestas a un cuestionario referido a la identificación de los factores de la ventaja competitiva que posee la universidad y la facultad.

En la investigación cuantitativa se aplica estadística inferencial donde se ha recolectado los datos por encuesta directa de los participantes de la muestra, para contrastar la hipótesis del estudio. La unidad de análisis es el ejecutivo en organizaciones dentro de la población del estudio: empresarios, académicos y profesionales que se desenvuelven a nivel de dirección en empresas, instituciones. El tamaño de muestra se ha determinado mediante estadística inferencial para pequeñas muestras con distribución normal, nivel de confianza de 95 $\%$, donde no se conoce la desviación estándar. El tamaño de muestra es de 25 ejecutivos.

\section{MARCO TEÓRICO}

\subsection{Capital creativo en el PCT}

El concepto PCT se ubica en el ámbito de la innovación, el cual es uno de los elementos primordiales para el desarrollo económico y social. En el ciclo de vida de los PCT se ha dado una evolución, que se dirige desde los parques tecnológicos de primera, segunda y tercera generación hacia los parques del conocimiento y la ciudad del conocimiento.

En la naciente Sociedad de la Información y el Conocimiento SIC, la innovación es el proceso que aplica el conocimiento creado y desarrollado dentro de los grupos humanos altamente capacitados que yacen en la Academia constituyendo la nueva clase creativa o del talento humano. La clave futura de la competitividad es atraer y retener a esa clase creativa. El factor de producción más valioso lo está constituyendo el capital creativo de las organizaciones que se manifiesta como la investigación aplicada que lleva a la mejora en la calidad del trabajo, de la calidad de vida, de la seguridad, la cultura. La nueva inversión a desplegar y consolidar es la infraestructura del saber, de manera que el término adecuado no será parque tecnológico, parque científico, node de desarrollo u otro, sino, parque del conocimiento [15].

\subsection{Tipos de parques científicos}

El devenir de los PCT se manifiesta en su complejidad de funcionamiento [12]:
- Primera generación, es el parque científico primigenio con una fuerte filosofía de innovación y de avance científico donde se destaca el emprendedor académico. Se concibe un modelo lineal de investigación, desarrollo e innovación tecnológica. Los primeros parques surgen en Estados Unidos con la Universidad de Stanford en California y el triángulo de investigación en North Carolina.

- Segunda generación, es el parque de alta tecnología con una filosofía innovadora y pragmática enfocada en el mercado, ligado a una universidad o instituto de Investigación. Las empresas promueven este modelo con el objetivo de innovar productos y servicios con mínima investigación básica. El principal ejemplo es el Parque Tecnológico Westfalia de Alemania.

- Tercera generación, es el parque científicotecnológico que combina las filosofías anteriores y depende del avance científico y del mercado, conjuntamente. Se concibe bajo un modelo interactivo de innovación denominado de la triple hélice, donde la innovación se produce mediante procesos continuos de aprendizaje y contacto con el entorno con el concepto de nodos de innovación.

- Cuarta generación, o ciudad del conocimiento, donde la localización geográfica es una de las decisiones críticas. Tienden a ubicarse en espacios urbanos, donde se integran los conceptos de conocimiento con desarrollo económico y planeación urbana, algunos ejemplos relevantes son Metrotech en Nueva York, Centro para la Ciencia de la ciudad universitaria en Philadelphia, la ciudad del conocimiento Yachay en Ecuador.

\subsection{La tendencia en el perfil y alcance del PCT}

Las tendencias en el desarrollo de parques tecnológicos son:

- El parque tecnológico tiende a denominarse parque del conocimiento.

- Se instala alrededor de instituciones del conocimiento, en ciudades.

- Las instituciones del conocimiento se constituyen en el núcleo del parque.

- El parque se conforma en una red de edificios con un área central coordinadora.

- El parque promueve nuevas y mejores formas de desarrollo urbano. 
- El parque desarrolla sinergias a partir de la colaboración con instituciones nacionales y gobierno, los empresarios e industria, y la comunidad local.

\subsection{El perfil estratégico del Parque científico- tecnológico}

El perfil estratégico de los parques se define con los siguientes elementos:

- La orientación a la ciencia y al mercado, buscando responder las cuestiones científicas y resolver los problemas del entorno.

- La generación de ambientes de innovación y creación de nuevas áreas de conocimiento.

- Los factores de atracción a las empresas, priorizando a las personas y el talento humano por encima del capital financiero, como era la política anterior.

- La intensidad tecnológica de sus productos y servicios, mediante la modernización de sectores de industria tradicional.

- Los productos y servicios orientados a cubrir necesidades de formación de empresas (micro-empresas de personas, start ups de corporaciones, pin-offs de la universidad).

- La función de excelencia e innovación abierta mediante asociaciones bajo el modelo triple hélice (universidad, industria, gobierno) incorporando la posibilidad de un cuarto actor para plantear un modelo de mayor cobertura o cuarta hélice.

\section{DISEÑO COMPETITIVO DE UN PCT}

\subsection{Los factores competitivos bajo el modelo diamante del desarrollo}

El éxito de las naciones se sustenta en el contexto general que palanquea la implantación de las estrategias adecuadas para un sector industrial, un clúster o una empresa específica. La competitividad de naciones [9] depende de cuatro factores: 1) Condiciones de los factores o recursos, 2) Estructura de los sectores y rivalidad, 3) Condiciones de la demanda o sociedad, 4) Sectores afines y de apoyo o institucionalidad, como se muestra en la figura 1.

- Factor 1 o la oferta del PCT. Se refiere a las condiciones de los factores. Se consideran los factores productivos de las facultades, donde se analiza el contexto y su desenvolvimiento, la disponibilidad de académicos e investigadores con capacidad de creación de productos e innovaciones, desarrollo de estudios aplicados para resolver problemas empresariales. Se incluye la infraestructura en terrenos y edificios, con locales en Lima y San Juan de Lurigancho, laboratorios que son recursos físicos directos o la elaboración de procesos y productos a nivel beta que son recursos indirectos. Comprende la oferta de la UNMSM en sus facultades y centros de producción con potenciales productos a brindar al medio empresarial, a partir de los cuales se elabora las ventajas competitivas de la universidad y su PCT.

- Factor 2 o estructura y rivalidad industrial. Se refiere a los sectores industriales, clúster y su grado de desarrollo de competencia.

Figura 1. Modelo del diamante del desarrollo y PCT.

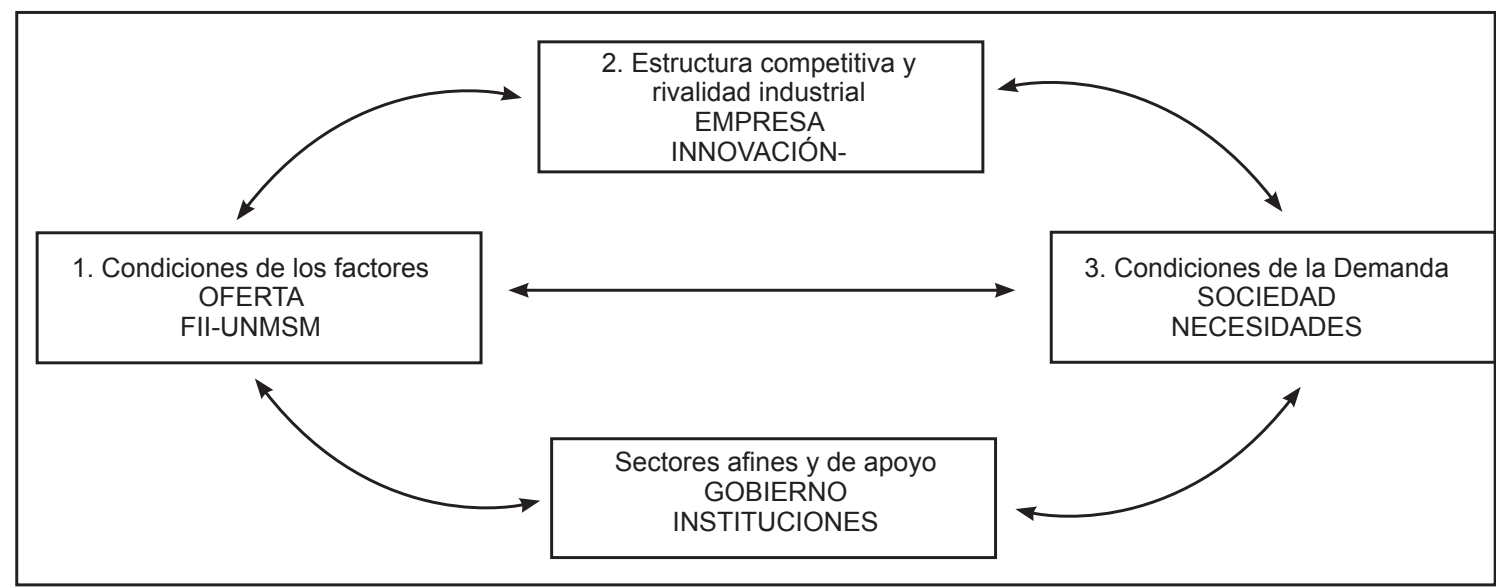


Dependiendo de la rivalidad competitiva, las empresas plantean sus objetivos $y$ estrategias para enfrentar el mercado, donde el beneficio competitivo del PCT se encuentra en su capacidad para atender las presiones empresariales por innovación y calidad, desarrollo de tecnología en producto y proceso. Su efecto se refleja en inversión, crecimiento productivo y exportaciones con valor agregado, obteniendo como resultado una industria competitiva a nivel internacional.

- Factor 3 o condiciones de la demanda. En este factor se consideran a las personas como oferta de fuerza de trabajo o como consumidores, sus necesidades, deseos, intereses, exigencias y el grado de satisfacción que esperan alcanzar a través de la oferta empresarial. La universidad cubre los requerimientos de formación y educación de las personas buscando conformar la clase creativa e innovadora que requiere la sociedad, la que ha de ser capaz de competir con éxito, con graduandos de contextos de mayor desarrollo industrial.

- Factor 4 o Sectores afines y de apoyo. Se refiere a la infraestructura conceptual del contexto cultural y su tradición industrial y la infraestructura física necesaria para que las empresas se interrelacionen entre sí, con sus proveedores y con sus mercados. Conforma el marco institucional donde se desenvuelven las empresas, su estabilidad genera confianza. Un componente relevante de la Universidad es su permanencia de medio millar de años.

\subsection{Sectores industriales, clústers y PCT}

Sector industrial es el agrupamiento de empresas clasificadas por la actividad central de la industria, para registro de la actividad macroeconómica [7]. Clúster es un grupo de empresas interconectadas que abarcan uno o más sectores productivos, próximos geográficamente, relacionadas entre sí por flujos comunes y complementarios de producción, mercado y distribución [11]. Ver la figura 2.

Esta figura muestra el aporte potencial que puede efectuar un PCT si se relaciona con los principales clúster productivos del país conformando una red de actividades que tiende a formarse y aglutinarse en torno a la explotación de un producto central o recursos naturales a fin de aumentar la productividad y eficiencia colectiva y generar sinergias y competencias distintivas.

En el país se han identificado seis grandes agrupamientos abarcando los mayores y más importantes sectores productivos, éstos son: a) Agrícola-alimenticio, b) Agrícola-industrial, c) Minero-metalúrgico, d) Petroquímico no Metálico, e) Pesquero, f) Textil y Confecciones.

Figura 2. Importancia del PCT en la creación y consolidación de clústers industriales.

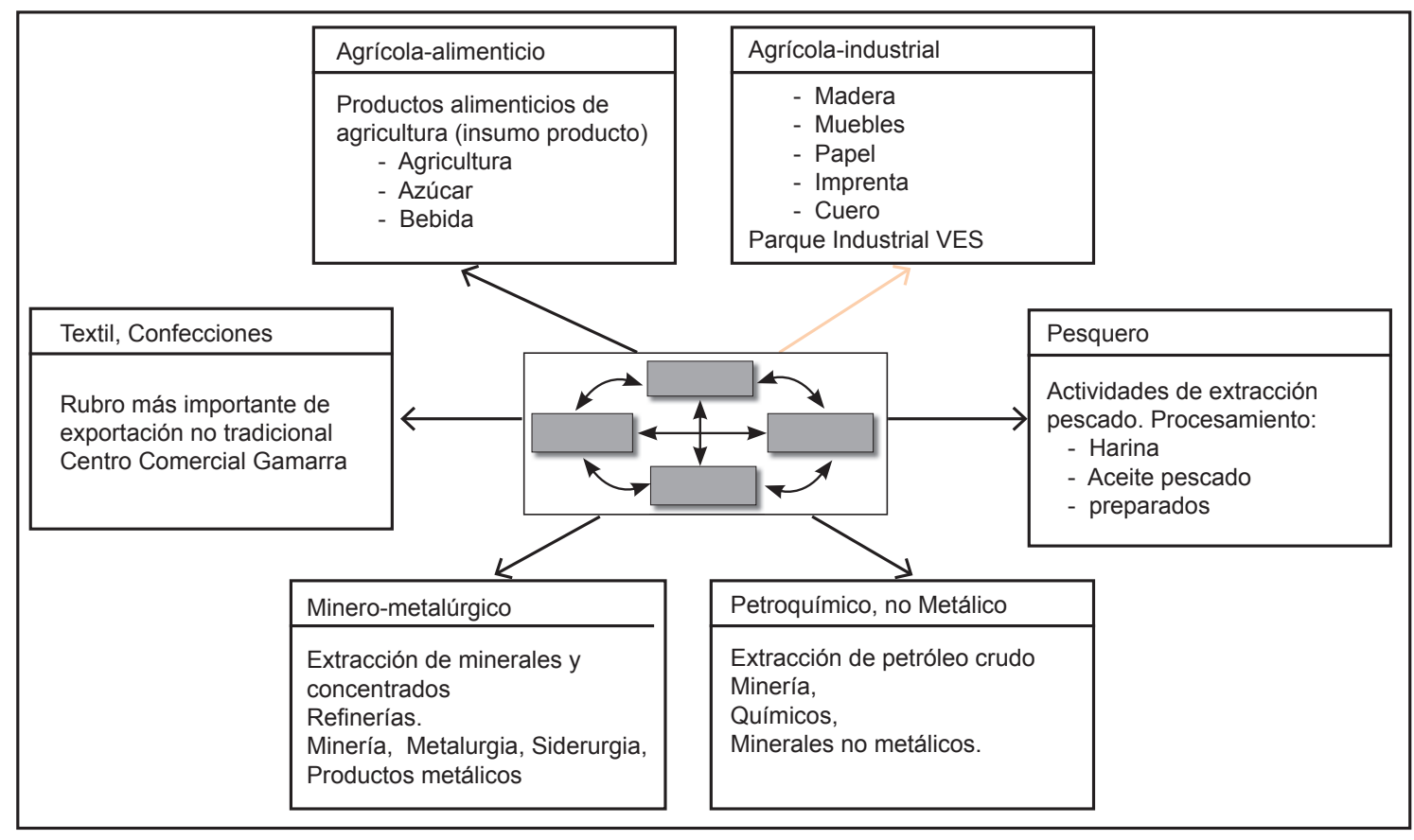


Su aporte sería sustancial para:

- Impulso de la innovación en productos y procesos.

- Aumento de la productividad individual y del grupo de empresas.

- Aumento de la capacidad competitiva ante las presiones de la globalización.

- Mayor capacidad de negociación en los mercados competitivos.

- Aumento de las expectativas de éxito ante el ingreso a nuevos mercados.

- Mayor influencia en mercados regionales protegidos.

- Generación sostenida y creciente de sinergias conjuntas.

\subsection{El modelo de negocio del PCT}

Un PCT requiere una estrategia competitiva para ofrecer un valor superior a las empresas, gobierno y sociedad, bajo el considerando que la competencia se ha extendido a todos los sectores de la sociedad incluyendo educación, investigación, proyección social, salud. El valor es la propuesta del PCT para satisfacer y superar las necesidades de investigación, innovación y desarrollo de la sociedad de manera eficiente. El valor del PCT es ofrecido por la universidad a los clientes (gobierno, empresas, sociedad, otras universidades), este valor social se plantea a partir del modelo de negocio del $\mathrm{PCT}$, el cual intenta describir la lógica económica de operación para distribuir el valor creado a los clientes con beneficio económico y social con un costo del empleo de recursos empleados cuando el

Tabla 1. Diseño de los elementos del modelo de negocio del PCT

\begin{tabular}{|c|c|c|c|}
\hline Elementos & Elementos & Criterios & Propuesta PCT \\
\hline Valor & $\begin{array}{l}\text { Propuesta de } \\
\text { valor }\end{array}$ & $\begin{array}{l}\text { ¿Productos y servicios que crean } \\
\text { valor para un segmento específico? } \\
\text { ¿Cuál es la razón para que prefieran } \\
\text { el PCT? }\end{array}$ & $\begin{array}{l}\text { Conocimiento básico y aplicado } \\
\text { Enlace necesidad-oferta empresarial } \\
\text { Grado de innovación } \\
\text { Desempeño. Diseño: producto } \\
\text { académico, marca, precio, costo. }\end{array}$ \\
\hline \multirow{2}{*}{$\begin{array}{l}1 \\
\text { Factores de la } \\
\text { oferta }\end{array}$} & Recursos claves & $\begin{array}{l}\text { Activos críticos para un eficaz modelo } \\
\text { de negocio: }\end{array}$ & $\begin{array}{l}\text { Recursos claves: intelectuales, } \\
\text { humanos, financieros, físicos }\end{array}$ \\
\hline & $\begin{array}{l}\text { Estructura de } \\
\text { costos }\end{array}$ & $\begin{array}{l}\text { ¿Cuáles son los costos más } \\
\text { importantes? ¿Qué recursos } \\
\text { y actividades claves son más } \\
\text { costosos? }\end{array}$ & $\begin{array}{l}\text { Estructura de costos } \\
\text { Estrategia de costo o de valor }\end{array}$ \\
\hline \multirow{2}{*}{$\begin{array}{l}2 \\
\text { Factores } \\
\text { competitivos de } \\
\text { las empresas }\end{array}$} & $\begin{array}{l}\text { Relaciones con } \\
\text { los clientes }\end{array}$ & $\begin{array}{l}\text { Tipo de relación que establecemos } \\
\text { con segmentos de clientes } \\
\text { específicos } \\
\text { contacto y negociaciones personales }\end{array}$ & $\begin{array}{l}\text { Adquisición, retención, salida, churn } \\
\text { de clientes } \\
\text { Incremento de participación } \\
\text { Relaciones con los clientes }\end{array}$ \\
\hline & $\begin{array}{l}\text { Flujos de ingresos } \\
\text { y egresos }\end{array}$ & $\begin{array}{l}\text { El flujo de financiamiento que } \\
\text { se genera de cada segmento de } \\
\text { mercado. }\end{array}$ & $\begin{array}{l}\text { Cada flujo con diferentes } \\
\text { mecanismos de valor y precios. } \\
\text { Tipos de flujos: por evento, } \\
\text { establecidos }\end{array}$ \\
\hline \multirow{2}{*}{$\begin{array}{l}3 \\
\text { Factores de la } \\
\text { demanda }\end{array}$} & $\begin{array}{l}\text { Segmentos de } \\
\text { mercado }\end{array}$ & $\begin{array}{l}\text { ¿Para quién se crea valor? } \\
\text { ¿Quiénes son los clientes más } \\
\text { importantes? }\end{array}$ & $\begin{array}{l}\text { Definir segmentos del mercado } \\
\text { empresarial y social } \\
\text { Academia, Empresa, Gobierno, } \\
\text { Sociedad }\end{array}$ \\
\hline & $\begin{array}{l}\text { Actividades } \\
\text { claves }\end{array}$ & $\begin{array}{l}\text { Core del negocio. Producción. } \\
\text { Solución de problemas. }\end{array}$ & $\begin{array}{l}\text { Investigación, innovación, formación } \\
\text { académica, consultoría empresarial } \\
\text { Básico, aplicado }\end{array}$ \\
\hline \multirow{2}{*}{$\begin{array}{l}4 \\
\text { Factores de } \\
\text { institucionalidad }\end{array}$} & Canales & $\begin{array}{l}\text { ¿Cómo se contacta y se comunica a } \\
\text { los segmentos de mercado } \\
\text { Opciones de alianzas }\end{array}$ & $\begin{array}{l}\text { Oferta de propuesta de valor } \\
\text { Medios de distribución } \\
\text { Acuerdos y asociaciones }\end{array}$ \\
\hline & Socios claves & $\begin{array}{l}\text { Red de proveedores y socios para } \\
\text { que el modelo funcione. } \\
\text { Compradores y vendedores }\end{array}$ & $\begin{array}{l}\text { Apoyo de gobierno } \\
\text { Alianzas con empresas } \\
\text { Alianzas con otras universidades }\end{array}$ \\
\hline
\end{tabular}


PCT genera, capta y distribuye el valor generado en diversas regiones [6)].

Sus elementos cubren los cuatro componentes del rombo de desarrollo, los que se presentan a continuación (ver tabla 1).

En el diseño del modelo de negocio, se plantea el foco de la estrategia de innovación. Dependiendo de las capacidades creativas e innovativas de la oferta de UNMSM, cada facultad ha de redefinir su potencial creador de conocimiento básico y aplicado, asociándolo con innovación tecnológica en producto, proceso, manufactura, maquinaria y equipo.

\section{DISEÑO DE LA ESTRATEGIA DE DESARRO- LLO DE PCT EN EL PAÍS}

\subsection{Definición de Parque Científico y Tecnológico}

Como se ha definido en estudios de investigación previos, "Parque Científico Tecnológico es una organización gestionada por profesionales especializados, cuyo objetivo fundamental es incrementar la riqueza de la comunidad promoviendo la cultura de la innovación y la competitividad de las empresas e instituciones instaladas en el parque o asociadas a él".

Un Parque Científico Tecnológico asume una perspectiva emprendedora a fin de estimular el surgimiento de nuevos negocios a través de la innovación tecnológica y para gestionar el flujo del conocimiento y tecnología generado o captado entre universidades, centros de investigación, empresas y mercados, de manera que se impulsa a creación de riqueza mediante la creación y crecimiento de organizaciones tecnológicamente innovadoras en productos y servicios. Los mecanismos para la captación, incubación, desarrollo y lanzamiento se ubican dentro de un espacio físico que contiene las instalaciones que apoyan para la ejecución de las tareas de innovación, transferencia de tecnología, creación de negocios, y que permite la conformación y consolidación de de un espíritu de crecimiento,

Figura 3. Efectos de un PCT y relación con la Academia e ingenierías de procesos.

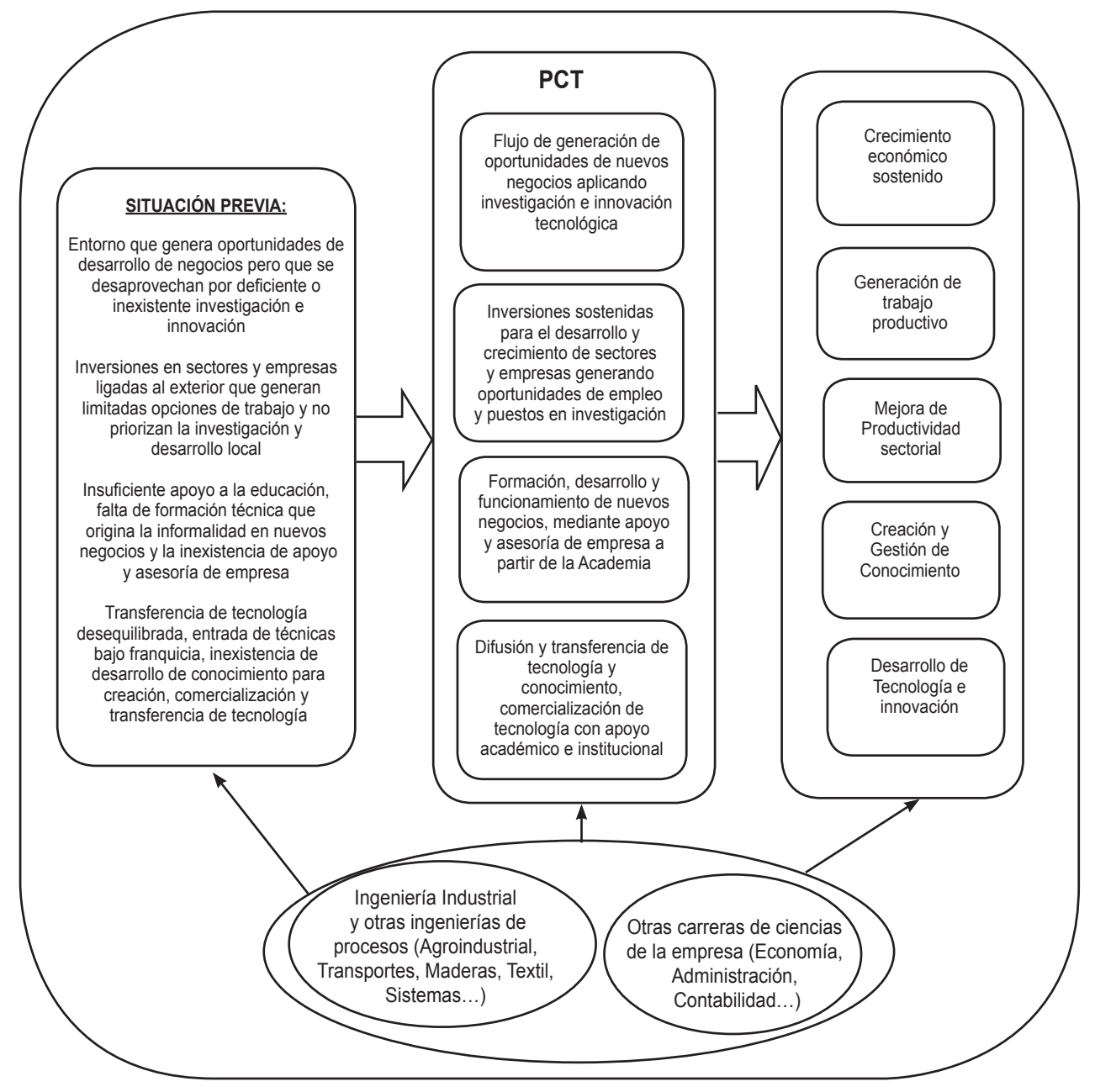


imaginación y emprendedurismo dentro de grupos humanos motivados y con elevadas expectativas de crecimiento personal.

La figura 3 presenta el rol integrador del PCT y los beneficios esperados de su funcionamiento. EI PCT se potencia con la intervención de la Academia, principalmente con su facultades integradoras y relacionadas a la tarea universitaria, la investigación y el desarrollo empresarial, como son Ingeniería Industrial e Ingeniería de Sistemas.

\subsection{Estrategia de desarrollo de Parque científico-tecnológico}

\section{Avances en el país}

En la actualidad, en el país, 16 universidades ya se encuentran implantando una incubadora de negocios, entre ellas la Universidad Nacional Mayor de San Marcos. La UNMSM es la primera universidad pública en lograr que su propuesta de incubadora de empresas presentada por la Facultad de Ingeniería Industrial sea reconocida por Start-Up Perú, el Ministerio de la Producción y el Fondo para la Innovación, la Ciencia y la Tecnología (FINCyT) [14].

Comparativamente, en diversos países de elevado y mediano desarrollo como España, India, Brasil, Chile, se han desarrollado parques tecnológicos gestionados conjuntamente por entes privados y gobiernos, con diferente grado de énfasis en su gestión y decisión [10].

\section{Parque científico-tecnológico y Academia}

Los parques industriales integrados a la Academia devienen en los parques científicos-tecnológicos. A nivel de gobiernos se les considera agentes primordiales en el sistema de investigación e innovación del país [8], su consolidación en una región eleva la atractividad para inversiones y propicia la creación e implantación de líneas de investigación científica y programas del desarrollo tecnológico.

Las razones para la creación de un PCT integrado a la Universidad, son:

- La implantación de proyectos PCT, significa una herramienta clave para el desarrollo y un estímulo para el crecimiento de negocios.

- EI PCT impulsa el desarrollo económico de las regiones y localidades donde se ubican.

- Incentiva la expansión de inversiones y promueve el surgimiento de iniciativas emprendedoras en una localidad.
- Revitaliza la dinámica económica en las empresas de la zona.

- Eleva las probabilidades de éxito de las empresas que se ubiquen en el parque industrial.

\section{Parque científico-tecnológico y UNMSM}

Dada la posición de la UNMSM, considerada la primera universidad del país, se debe llegar a hacia la comunidad empresarial, a través de las líneas de investigación redefinidas en las facultades de empresa, de manera que difunda y se perciba el potencial de la capacidad científico-técnica de la UNMSM, a través de la oferta de $1+D+i+e$, según lo requiere la dinámica de crecimiento económico de la reciente década pasada. Se han de identificar las necesidades de investigación y tecnológicas de los mayores sectores productivos [4], para el desarrollo, aplicación y comercialización de las tareas $\mathrm{I}+\mathrm{D}+\mathrm{i}+\mathrm{e}$ generados en la UNMSM.

El Vicerrectorado de Investigación ha de consolidar a la UNMSM en el ambiente empresarial mediante contratos entre universidad y empresa. Mediante una Oficina de Transferencia de Investigación se buscaría que los resultados de investigaciones realizadas en la UNMSM, trasciendan el ámbito del laboratorio.

\section{Parque científico-tecnológico y facultades de Ingeniería}

Excluyendo las ingenierías tradicionales enfocadas en manejo de materiales, existen dos grandes profesiones de las cuales se han derivado diversas carreras profesionales enfocadas en empresa. Las ingenierías enfocadas en resultados y empresa son, básicamente, dos: Ingeniería Industrial e Ingeniería de Sistemas, ambas existentes en la UNMSM, cubriendo otras escuelas enmarcadas dentro de la visión de desarrollo empresarial.

Estas facultades, por su carácter integrador, engloban actividades relacionadas a la misión de un PCT, como la promoción de cursos con el objetivo de creación de conocimiento y posibilidad de negocio y transferencia de tecnología, posibilidad de agrupar equipos interdisciplinarios que desarrollen investigaciones, posibilidad de desarrollar expertise en spin-off y patentes.

El Spin-off se define como la empresa nueva conformada por miembros del centro de investigación de la universidad, con la finalidad de transferir conocimiento y brindar a los investigadores la posibilidad de llevar a la práctica los resultados de sus proyectos. 
5.3. Elementos estratégicos del diseño estratégico del PCT

\section{Los elementos de competitividad}

\section{Las áreas temáticas de un PCT}

Un parque científico tecnológico debe enfocarse en las siguientes áreas:

- Tecnologías de avanzada en informática, software.

- Tecnologías en sectores tradicionales y de alta rentabilidad: minería, pesquería.

- Tecnologías para gestión y manejo del medio ambiente y reservas ecológicas.

- Tecnologías en industrias de servicios

- Tecnologías en sectores emergentes: agroindustria, textil, artesanía.

\section{Las variables estratégicas de competitividad nacional}

Los elementos competitivos de un PCT, se resumen a continuación:

- Ubicación geográfica. Por su efecto en costos, el PCT se ubica cercano las fuentes de recursos o cercano a los mercados de consumo de conocimiento, incluyendo cercanía a vías de comunicación como puertos, aeropuertos, carreteras.

- Infraestructura de apoyo a investigación. Comprende los servicios básicos de energía eléctrica, sanitarios, comunicaciones, seguridad, administración.

- Administración: en los parques tecnológicos existen profesionistas especializados en la gestión y administración de servicios y en atender necesidades de las empresas que residen en ellos.

- Apoyo del gobierno: es frecuente que los parques tecnológicos reciban apoyo del gobierno, mediante la creación de un fondo, programas especiales o fideicomisos.

\section{Las capacidades distintivas de la competitividad de un PCT}

Un PCT bajo la gestión de la universidad, debe abarcar las siguientes capacidades estratégicas:

\section{Capacidad de innovar}

Un PCT, sustentado en la academia, debe poseer dos características fundamentales:
- Capacidad de detectar y promover la imaginación y la creatividad de sus integrantes.

- Capacidad de innovación y desarrollo de productos físicos y mentales.

\section{Capacidad de crear sinergias}

Un PCT promueve el desarrollo económico y la competitividad de las regiones y ciudades [13], mediante:

- Creación de nuevas oportunidades de negocio y añadir valor para madurar las empresas

- Fomento del espíritu empresarial y la incubación de nuevas empresas innovadoras

- Generación de empleos basada en conocimientos

- Construcción de espacios atractivos para los trabajadores del conocimiento

- Creación y mejora de sinergia entre universidades y empresas

\section{Capacidad de promoverse por su oferta}

La oferta investigativa de la universidad es su catálogo $\mathrm{I}+\mathrm{D}+\mathrm{i}+\mathrm{e}$.

- El catálogo I+D+i+e de la universidad es una herramienta esencial para los grupos de investigación, que han de utilizar su potencial y ponerlo en práctica en la empresa, y para la oficina de transferencias a fin que relaciones las líneas de investigación con los requerimientos tecnológicos.

- El catálogo I+D+i+e se debe manejar con criterio de eficiencia económica, además se mantiene objetivos de mejora continua y adaptación al ámbito empresarial, con compromiso en el diseño de procesos y servicios para satisfacer las necesidades de los clientes empresariales.

\section{Capacidad de desarrollar entregables}

Un PCT representa un servicio universitario que está compuesto por distintas áreas que responden a las necesidades de creación y transferencia de tecnología, innovación e investigación y difusión del conocimiento que ofrecen los grupos de investigación. EI PCT recibe las ofertas y demandas de colaboración, ofrece asesoramiento específico, negocia y realiza trámites para implementar acuerdos.

Los entregables del PCT son:

- invenciones generadas en los proyectos PCT.

- patentes, para el registro de la propiedad industrial, 
- contratos, a través de la cual se intermedia entre la empresa y el investigador;

- asesoría en negocio, económico, gestión para los grupos que participen en los proyectos.

\section{Capacidad de desarrollar patentes}

Un área de patentes de la UNMSM gestiona las solicitudes y orienta y apoya al investigador en la tramitación y registro de sus inventos. La UNMSM debe elaborar un catálogo $\mathrm{I+D}+\mathrm{i}$ con información de todos los proyectos y grupos de investigación, acercando la investigación universitaria a las empresas.

\subsection{La estrategia de desarrollo del PCT}

Para elegir el modo de implantar un PCT, se debe considerar las fases por las que debe transitar en su evolución hacia estados superiores [3], considerando la situación actual, los actores y decisores involucrados el objetivo final que es un PCT que aporte al desarrollo, según el esquema mostrado en la figura 4.

Las características de un PCT, conformado por academia, empresa y estado, son las siguientes:

- Los actores son académicos investigadores y emprendedores.

Figura 4. Diseño del desarrollo de PCT con Academia (UNMSM), Gobierno, Empresa.

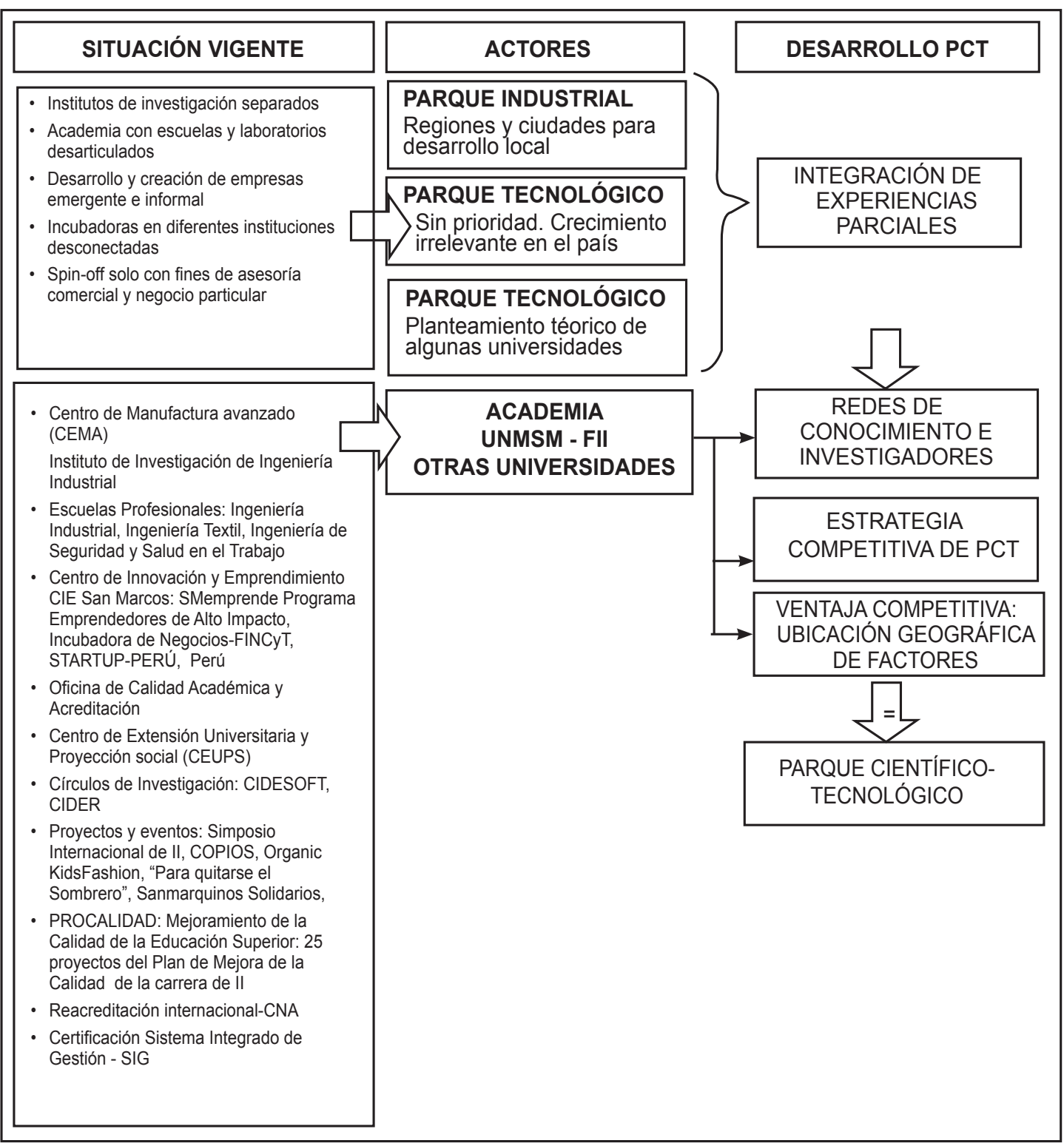


- Posee ventajas competitivas para atraer a actores de otras regiones [1].

- Posee recursos competitivos para establecer capacidades formativas y redes de conocimiento.

- Gestión y funcionamiento eficaz para el adecuado flujo de conocimiento entre los actores.

- El espacio geográfico, recursos y factores son la base de la generación de conocimiento.

- Enlaza redes de investigadores con las organizaciones y la infraestructura para el desarrollo de las innovaciones.

\subsection{El modelo de gestión para consolidar un PCT}

\section{Gestión y organización del PCT}

En general, se consideran cinco modelos alternativos para la organización y la gestión de un PCT, las que dependen de la visión de los decisores, de los recursos disponibles y del objetivo por el cual se implanta el PCT.

\section{Estas opciones son:}

- Gestión por una universidad, como el caso de parques en México.

- Gestión por el gobierno central.

- Gestión por un ente autónomo con funcione especializadas.

- Gestión por promotores privados sin intervención estatal ni universidad.

- Gestión por organizaciones no gubernamentales con fines sociales.

\section{Las funciones básicas del PCT}

Además del propósito de su implantación, un PCT desarrolla cuatro tipos de funciones básicas:

- Adopción y transferencia de tecnología. A partir de la adopción de la innovación, se realiza la difusión y la transferencia de tecnología a nivel sectorial-empresarial y a nivel geográfico, para su adaptación y explotación.

- Desarrollo de innovaciones. Mejorando la tecnología tradicional y generando tecnología de avanzada.

- Creación de factores competitivos. Que permita un entorno académico, científico y de investigación donde se ubiquen geográficamente los factores de competitividad.
- Incubadora de negocios. Para la creación, lanzamiento y marcha normal de empresas basadas en tecnología.

\section{Las funciones de apoyo del PCT}

Las funciones generales para el funcionamiento del PCT son:

- Gestionar espacios para el desarrollo de actividades de centros de investigación o empresas de servicios, planta manufacturera, emprendimiento, innovación tecnológica, inicio e incubación de nuevos negocios.

- Brindar servicios de información y consultoría para establecimiento de organizaciones de negocio, desde las alianzas, el financiamiento, tutoría, diseño de proyectos y planes de negocio, capacitación de tipo formal.

- Brindar servicios de apoyo y servicios básicos para el funcionamiento de la actividad de investigación e innovación, incluyendo gestión de proyectos, estudio de mercado, transferencia de tecnología.

- Fomento de nuevos negocios, actividades de marketing y la promoción, para el desarrollo empresarial.

\section{RESULTADOS DE LA INVESTIGACIÓN EMPÍ- RICA}

\section{Corroboración de hipótesis}

En el análisis de resultados, para un nivel de significación de 0.05 , el F calculado es superior al F teórico $(28.583>2.70)$. Entonces, se rechaza la hipótesisnulaqueafirmaquela Facultad deIngeniería Industrial no posee ventajas competitivas que se plasme en capacidad de investigación y desarrollo dentro de un proyecto PCT. Alternativamente, se corrobora la hipótesis alterna, por lo tanto, la Facultad de Ingeniería Industrial posee ventajas competitivas que conforman su capacidad de investigación y desarrollo aprovechables dentro de un proyecto de Parque Científico-Tecnológico.

La hipótesis $\mathrm{H}_{1}$ no rechazada señala que el diseño de la estrategia competitiva se ha de dirigir a los factores clave para la creación de conocimiento y la innovación, esto es, debe enfocarse en el factor 1 de condiciones de los factores $u$ oferta creativa de la FII o conocimiento académico e investigativo orientado al desarrollo de innovaciones para asentar las ventajas competitivas en la operación de empresa, con sentido pragmático y utilitario (ver figura 3). 
Figura 5. Corroboración de hipótesis.

H1: Se rechaza hipótesis nula: el diseño de la estrategia competitiva se ha de dirigir a los factores clave: la oferta creativa de la UNMSM orientada hacia la operación de empresa, con sentido práctico y utilitario.

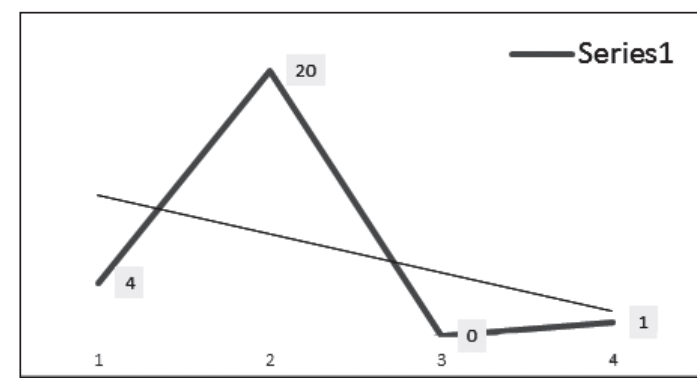

Se busca verificar la hipótesis, sobre que el PCT representa el surgimiento de potenciales ventajas competitivas que han de permitir una superior capacidad de investigación, creación de conocimiento y mayor influencia de la universidad en el ambiente social y económico.

\section{Aporte empírico sobre estrategia de PCT}

Se estima que los resultados obtenidos serán válidos en la medida que permitan el planteamiento de contenido teórico para entender la realidad y para predecir empíricamente a elementos factuales de la realidad de los parques científico tecnológicos.

Con el esquema del diamante del desarrollo, los ingenieros consideran que la formación de la estrategia de la FII debe enfocarse en los factores de oferta y demanda (conocimiento, investigación de académicos) más requerimientos de la sociedad (necesidades, proyección) como se presenta en la figura 4 que describe el sesgo decisional del grupo muestral.

Figura 6. Sesgo hacia factores o hacia operatividad

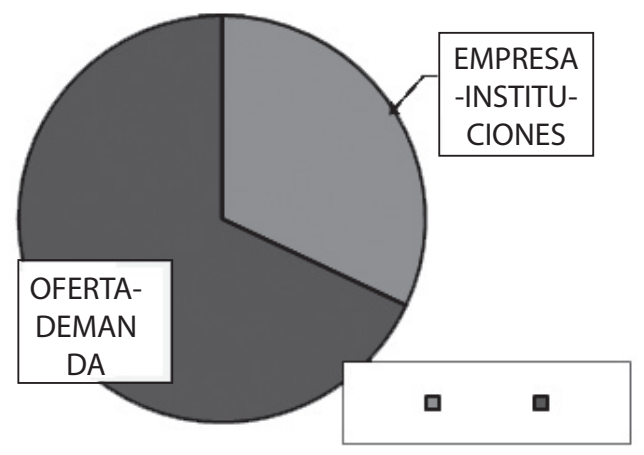

En la figura 5 se presenta el modelo de Pareto, que define el orden de preferencia de los factores. El factor 1 oferta de factores de la academia y el factor 2 demanda de innovación y competitividad son los que manifiestan la mayor preferencia. Esta elección guarda congruencia con las tendencias establecida en los antecedentes, ya que la academia ofrece lo que considera su mayor ventaja competitiva (conocimiento e investigación) la que debe ser orientada hacia aspectos prácticos de la competitividad de las empresas, como es resolver problemas e innovar en productos y procesos.

Figura 7. Pareto de factores del diamante del desarrollo

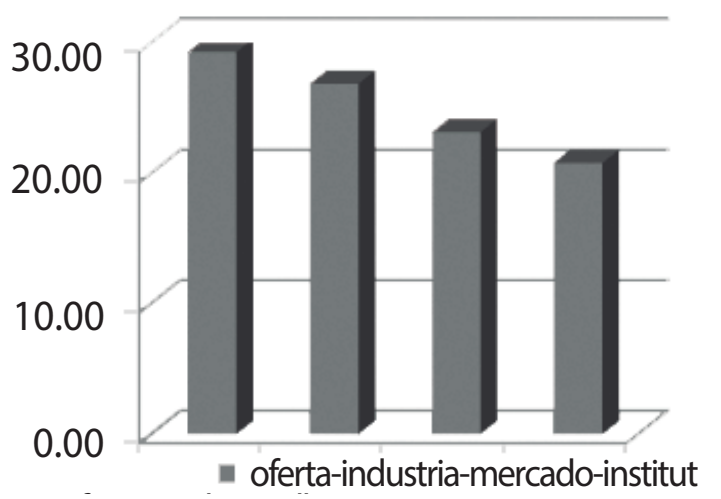

\section{Discusión de la ventaja competitiva de un PCT en la UNMSM}

La ventaja competitiva de un parque científico tecnológico se construye a partir de los hallazgos, derivados de la pregunta de investigación y de la corroboración de la hipótesis de trabajo, donde se plantea que las ventajas competitivas de un PCT en la FII deriva de la apropiada priorización de los elementos del rombo del desarrollo, los que se refieren al potencial de recibir empresas e inversiones con fines de investigación e innovación y luego proyectarlo hacia la sociedad en los siguientes ítems:

- Parque científico-tecnológico y el país. Urbano, en regiones.

- Parque científico-tecnológico y Academia. Liderado por UNMSM en alianza con otras.

- Parque científico-tecnológico e Ingeniería. FII como enlace de facultades y empresa. 


\section{El modelo dinámico de Parque Científico y Tecnológico}

Según el modelo de la triple hélice, el PCT se potencia con la intervención de la Academia, el desarrollo empresarial y el gobierno que busca crecimiento y empleo [5]. Desde la perspectiva emprendedora un parque estimula y gestiona conocimiento y tecnología entre universidades, centros de investigación, empresas y mercados, impulsando la creación y el crecimiento de empresas innovadoras con variados mecanismos de incubación (figura 6).

\section{REFERENCIAS BIBLIOGRÁFICAS}

[1] Banco Mundial (2008). "International Good Practice For Establishment Of Sustainable It Parks. Review of Experiences in Select Countries, Including Three Country Case Studies: Vietnam, Russia \& Jordan." Extraído el 14-04-12 desde: http://www. infodev.org/en/ Document.557.pdf

[2] Castells, Manuel (1989). La ciudad informacional: tecnologías de la información, reestructuración económica y el proceso urbano regional. Alianza Editorial. Madrid, España.

Figura 8. El modelo PCT y el enfoque triple hélice

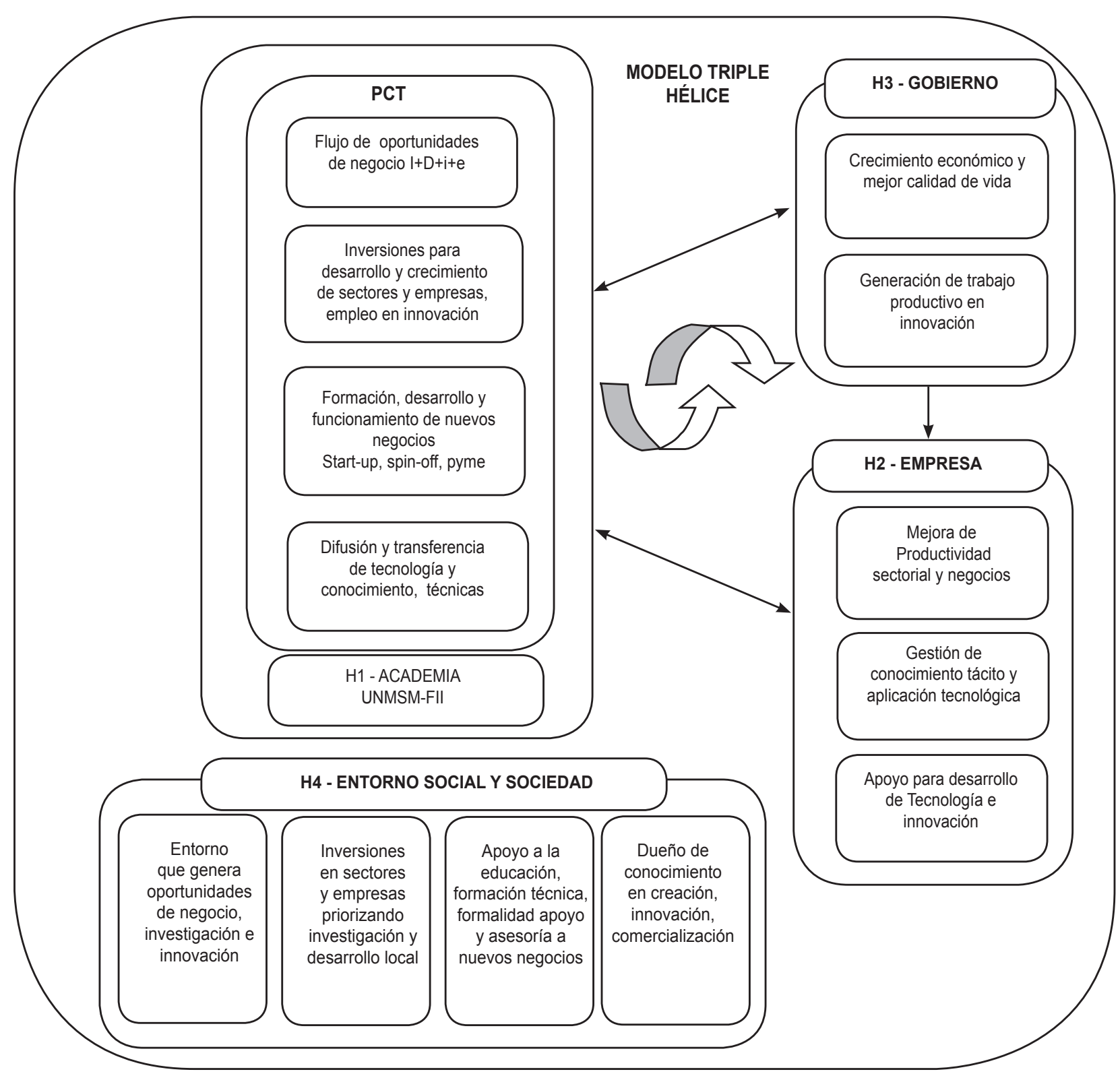


[3] DPN (2011) "Plan Nacional de Desarrollo: Prosperidad para Todos (2010-2014) - Capítulo III-Crecimiento sostenible y competitividad.. Pág. 48 - 248. Extraído el 14-08-12 desde: http://www.dnp.gov.co/portalweb/linkclick.

[4] Kista Science City. (2010). "Kista Science City". Extraído el 19-5-12 desde: http://www.kista. com/adimo4/site/kista/web/default.

[5] Leydesdorff (2006). "The Knowledge-Based Economy and the Triple Helix Model". Extraído el 10-7-12 desde: http://www.leydesdorff.net/ arist09/arist09.pdf.

[6] Mayer, Heike (2007). "What Is the Role of the University in Creating a High-Technology Region?". Journal of Urban Technology, 14:3, 33 - 58. Extraído el 01-12-2013 desde: http:// dx.doi.org/10.1080/10630730801924225

[7] Naciones Unidas (2009). Clasificación Industrial Internacional Uniforme de todas las actividades económicas (CIIU). Informes estadísticos Serie M, No. 4/Rev. 4. Nueva York.

[8] National Research Council. (2009). "Understanding Research and Technology Parks.Global Best Practice, Report of Symposium National Academies Press". Extraído el 11-10-12 desde: http://www.nap. edu/catalog.php.

[9] Porter, Michael (2008). Ser Competitivo. Edición actualizada y aumentada. Harvard Business Press y Ediciones DEUSTO, España.
[10] Porter, Michael (2006). Estrategia y ventaja competitiva. Ediciones DEUSTO, España.

[11] Torres Z., Jorge (2003). Clusters de la industria en el Perú. Documento de trabajo 228. PUCP. Extraído el 12-08-2013 desde: http:// departamento.pucp.edu.pe/economia/images/ documentos/DDD228.pdf.

[12] Ugalde y Sáenz (2012). Los parque tecnológicos urbanos como instrumentos de regeneración del territorio: el caso de Zorrozaurre. Extraído el 15-11-2013 desde: http://www.ciccp.es/biblio digital/Urbanismo_//congreso/pdf/050103.pdf

[13] Universia (2013). Competitividad en el Perú: Diagnóstico, sectores a priorizar y lineamientos a seguir para el período 2011-2016. Universia del 11-11-2010). Extraído el 18-08-2013 desde: http://gcg.universia.net/pdfs_revistas/ articulo_179_1301298918203.pdf

[14] UNMSM-FII (2014). Revista Industrial Edición de aniversario. Año 4, N 1, diciembre 2014. Perú.

[15] Vaccarezza, Leonardo (2011). "Ciencia, Tecnología y Sociedad: el estado de la cuestión en América Latina" en Revista do Observatório do Movimiento pela Tecnologia social da America Latina. Brasil. Extraído el 14-11-2013 desde: http://seer.bce.unb.br/index.php/cts/ article/view/3841/3298 